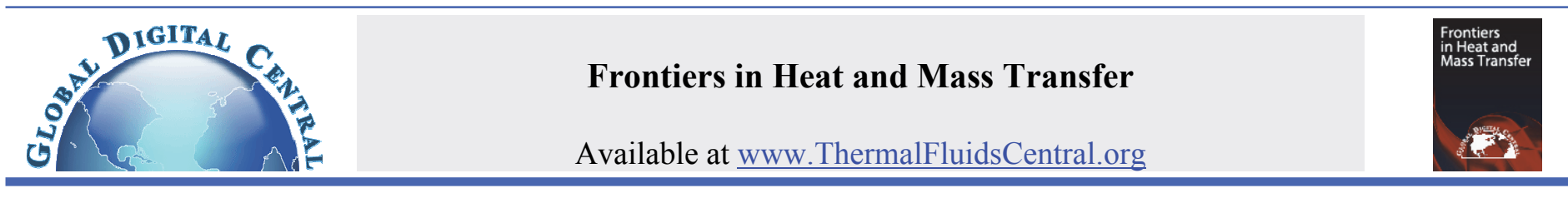

\title{
COMPUTATIONAL FLUID DYNAMICS SIMULATION OF THE THERMAL UNIFORMITY IN CATALYTIC MICRO-COMBUSTORS
}

\author{
Junjie Chen*, Wenya Song, Deguang Xu \\ School of Mechanical and Power Engineering, Henan Polytechnic University, Jiaozuo, Henan, 454000, China
}

\begin{abstract}
The combustion and heat transfer characteristics of hydrogen-air or methane-air mixtures in catalytic micro-combustors were studied numerically to assess the impact of wall thermal properties and key operation parameters on the thermal uniformity. A two-dimensional computational fluid dynamics (CFD) model was developed with detailed hetero-/homogeneous chemistry, heat conduction within the solid wall, surface radiation heat transfer, and external heat losses. Parametric studies were carried out to investigate the effect of wall thermal conductivity, feed composition, and flow rate on the thermal uniformity during highly exothermic catalytic reactions. Comparisons of hydrogen- with methane-air systems were made. Based on these insights, an overall energy balance analysis was performed in terms of enthalpy loss. It was shown that the wall thermal properties strongly affect the thermal uniformity, but little impact on the extinction limit and conversion. The feed composition and flow rate have a significant impact on the operating temperature, but only a moderate effect on the thermal uniformity. Most of the enthalpy released by the exothermic reaction is lost to the surroundings under certain conditions, although this energy exchange becomes less efficient as the flow rate increases. This feature is beneficial for heat-exchanger applications.
\end{abstract}

Keywords: Micro-combustion; Thermal management; Flame stability; Thermal uniformity; Catalytic combustion; Computational fluid dynamics.

\section{INTRODUCTION}

There has been increasing interest in utilizing the high energy density of fuels in portable power generation systems (Kaisare and Vlachos, 2012). Micro-combustors are emerging as a powerful tool for portable power generation devices. Due to the large energy density of hydrocarbons, micro-combustion may eventually replace expensive and environmentally non-benign lithium batteries in portable electronics devices (Ju and Maruta, 2011). Furthermore, due to the inherently higher heat-transfer coefficients, micro-combustors can be efficient heat sources for endothermic reactions, such as ammonia decomposition (Chiuta et al., 2013) and steam reforming (Jeon et al., 2014), in integrated micro-devices for the production of hydrogen for fuel cell applications. Homogeneous flames are typically quenched when confined in the sub-millimeter range because of thermal and radical quenching at combustor walls. While homogeneous micro-combustors are feasible to operate, their operation is restrictive. Increasing surfaceto-volume ratios render catalytic combustion the preferred route compared to pure homogeneous combustion. Catalytic combustion can significantly extend the classical flammability limits, and can result in faster effective reaction rates, more stable operation, and process intensification (Norton et al., 2006; Tolmachoff et al., 2015). Additionally, catalytic micro-combustors have the potential to operate at significantly lower temperatures with significant heat losses (Federici et al., 2006; Karagiannidis et al., 2007), and their ignition temperatures are lower (Appel et al., 2002; Norton et al., 2004). Furthermore, catalytic systems are typically easier to start, and can be designed with very simple geometries and no moving parts, which dramatically simplifies miniaturization and reduces cost (Federici et al., 2006).

Thermal management may play a vital role in micro-combustors (Haber et al., 2012). The effective thermal management of the released heat can improve the efficiency of portable power generation systems so that their energy density exceeds that of traditional lithium batteries (Federici et al., 2006; Rana et al., 2014). In addition, thermal management plays an important role in minimizing heat loss, mitigating thermal quenching, and increasing device performance (Di Stazio et al., 2016; Miesse et al., 2004). Moreover, thermal management is the key to successful operation, especially micro-combustors integrated with other components or devices such as small-scale fuel processing or reforming systems (Holladay and Wang, 2015; Merotto et al., 2016). For example, for coupled micro-combustor-thermoelectric devices, it should be an important design goal to achieve temperature uniformity (Federici et al., 2006). Furthermore, for commercial applications, thermal management will be crucial to deliver cost-effective and durable devices (Walther and Ahn, 2011). In order to improve combustion stability and thermal efficiency, various efforts have been paid on the optimization of thermal management. One thermal management strategy to overcome heat losses is the utilization of excess enthalpy micro-combustors, in which the hot exiting products are used to exchange heat with the incoming cool reactants (Shirsat and Gupta, 2011). Increased stability results from the transverse heat exchange between the incoming reactants and the hot products (Shirsat and Gupta, 2011). Heat recirculation is an example of these excess enthalpy micro-combustors because it can significantly enhance combustor stability and can greatly reduce heat loss (Bagheri and Hosseini, 2015), and the Swiss-roll configuration is such an effective heat recirculation device (Kim et al., 2005; Kim et al., 2007). Another thermal management strategy to increase conversion is the utilization of spatial catalyst segmentation (Chen et al., 2008).

For many applications, isothermal or near-isothermal operation is favorable, and the degree of thermal uniformity can adversely or favorably affect the performance (Jeon et al., 2013; Shigarov et al., 2009). Temperature non-uniformity and problems associated with hot

*Corresponding author.Email: comcij@163.com. 
spots at the macro-scale are well recognized (Boger and Heibel, 2005; Groppi and Tronconi, 2005). However, less work has been devoted to the thermal uniformity in micro-combustors. Thermal uniformity is often assumed because of small characteristic length scales and consequently high transport rates. However, this assumption is not necessarily correct, especially for well-insulated micro-combustors with fast reaction chemistries. Typically, micro-combustors have one dimension in the sub-millimeter range and the remaining dimensions are in the meso-scale or macro-scale range. Therefore, thermal uniformity may be achieved in the micro-scale dimension, but significant thermal gradients may exist in the remaining dimensions. Both of these conditions can present challenges for efficient conversion, material selection, and integration with other components or devices.

In this work, the combustion and heat transfer characteristics of hydrogen-air or methane-air mixtures in catalytic micro-combustors were studied using a two-dimensional CFD model with detailed chemistry and transport. Parametric studies were performed to investigate the effect of wall thermal conductivity on the thermal uniformity during highly exothermic catalytic reactions. Additionally, the effects of feed composition and flow rate on the combustor temperature and thermal uniformity were delineated. Finally, an overall energy balance analysis was performed in terms of enthalpy loss. The main objective is to assess the impact of wall thermal properties and key operation parameters on the thermal uniformity. The findings of the present work could further facilitate the design of catalytic microcombustors and address combustor thermal management issues.

\section{NUMERICAL MODELS AND SIMULATION APPROACH}

\subsection{Geometric model}

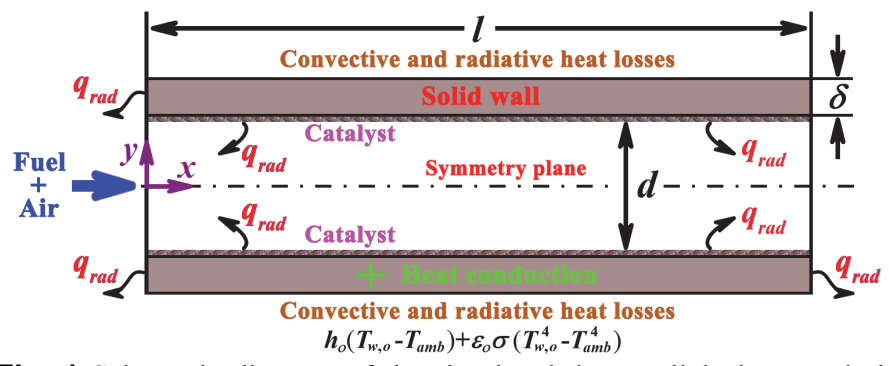

Fig. 1 Schematic diagram of the simulated the parallel plate catalytic micro-combustor. Due to symmetry, only half of the combustor is simulated. Note that the $y$ dimension is scaled by factors of 2 for ease of visualization.

Fig. 1 shows a schematic of the catalytic micro-combustor modeled in this work. The combustor consists of two parallel plates of thickness $\delta$ and length $l$, separated by a gap size $d$ between them. The nominal combustor dimensions used in this work are $d=0.8 \mathrm{~mm}, \delta=0.2 \mathrm{~mm}$, and $l=8.0 \mathrm{~mm}$. The parallel plate geometry implies that the third dimension (width) of the combustor is much larger than the gap size. Flow rates $Q$ reported throughout consider a combustor width (in the third dimension) of $10.0 \mathrm{~mm}$, and are given to provide a sense for real experiments. The parallel plates are coated with platinum catalysts. Due to the aspect ratio, the combustor is modeled as a two-dimensional system and the plane of symmetry between the two plates allows simulations of only half of the combustor. Fuel-air mixtures of varying concentrations are fed into the combustor at room temperature, and hydrogen and methane fuels are examined.

\subsection{Mathematical model}

The following assumptions are made: the flow is laminar, the pressure drop is negligible, and gases follow the ideal gas law. The heat transfer in the form of surface radiation within catalytic micro-combustors can have an important impact on the energy balance and flame stability, and thus the radiation exchange between channel wall surface elements is considered in this work. A two-dimensional steady state model is employed using the commercial CFD software package ANSYS FLUENT $^{\circledR}$ Release 6.3 (Fluent Inc., 2006) incorporates with the detailed homogeneous and heterogeneous reaction schemes in CHEMKIN (Kee et al., 1996) and Surface-CHEMKIN (Coltrin et al., 1996) format to simulate the laminar flow in the catalytic microchannel. The governing equations for a steady, laminar, twodimensional reactive flow with homogeneous and heterogeneous reactions in Cartesian coordinates are given as follows:

Continuity equation:

$\frac{\partial(\rho u)}{\partial x}+\frac{\partial(\rho v)}{\partial y}=0$.

Momentum equations:

$\frac{\partial(\rho u u)}{\partial x}+\frac{\partial(\rho v u)}{\partial y}+\frac{\partial p}{\partial x}-\frac{\partial}{\partial x}\left[2 \mu \frac{\partial u}{\partial x}-\frac{2}{3} \mu\left(\frac{\partial u}{\partial x}+\frac{\partial v}{\partial y}\right)\right]-\frac{\partial}{\partial y}\left[\mu\left(\frac{\partial u}{\partial y}+\frac{\partial v}{\partial x}\right)\right]=0$, (2)

$\frac{\partial(\rho u v)}{\partial x}+\frac{\partial(\rho v v)}{\partial y}+\frac{\partial p}{\partial y}-\frac{\partial}{\partial x}\left[\mu\left(\frac{\partial v}{\partial x}+\frac{\partial u}{\partial y}\right)\right]-\frac{\partial}{\partial y}\left[2 \mu \frac{\partial v}{\partial y}-\frac{2}{3} \mu\left(\frac{\partial u}{\partial x}+\frac{\partial v}{\partial y}\right)\right]=0$.

Energy equation:

$\frac{\partial(\rho u h)}{\partial x}+\frac{\partial(\rho v h)}{\partial y}+\frac{\partial}{\partial x}\left(\rho \sum_{k=1}^{K_{g}} Y_{k} h_{k} V_{k, x}-\lambda_{g} \frac{\partial T}{\partial x}\right)+\frac{\partial}{\partial y}\left(\rho \sum_{k=1}^{K_{g}} Y_{k} h_{k} V_{k, y}-\lambda_{g} \frac{\partial T}{\partial y}\right)=0$.

Gas phase species equation:

$\frac{\partial\left(\rho u Y_{k}\right)}{\partial x}+\frac{\partial\left(\rho v Y_{k}\right)}{\partial y}+\frac{\partial}{\partial x}\left(\rho Y_{k} V_{k, x}\right)+\frac{\partial}{\partial y}\left(\rho Y_{k} V_{k, y}\right)-\dot{\omega}_{k} W_{k}=0, k=1, \ldots, K_{g}$.

In the above equations, $\rho$ is the density of gas, $u$ and $v$ are the streamwise and transverse velocity component, $x$ and $y$ are the streamwise and transverse coordinates, $p$ is the pressure, $\mu$ is the dynamic viscosity of gas, $K_{g}$ is the total number of gaseous species, $T$ is the temperature, $h$ is the total enthalpy of gas mixture, $Y_{k}, h_{k}, \dot{\omega}_{k}$, and

$W_{k}$ are the mass fraction, chemical enthalpy, homogeneous molar production rate, and molecular weight of $k$-th gaseous species respectively, $V_{k, x}, V_{k, y}$ are the $x$ - and $y$-components of the $k$-th gaseous species diffusion velocity, and $\lambda_{g}$ is the thermal conductivity of gas.

Species diffusion velocities $\vec{V}_{k}$ are computed using mixture average diffusion, including thermal diffusion for the light species $\mathrm{H}$ and $\mathrm{H}_{2}$ (Kee et al., 1998):

$\vec{V}_{k}=-D_{k, m} \nabla\left[\ln \left(Y_{k} \bar{W} / W_{k}\right)\right]+\left[D_{k}^{T} W /\left(\rho Y_{k} \bar{W}\right)\right] \nabla(\ln T)$.

where $D_{k, m}$ and $D_{k}^{T}$ are the mixture-average and thermal diffusion coefficient of the $k$-th gaseous species, respectively, and $\bar{W}$ is the average molecular weight of gas mixture.

The ideal gas and caloric state laws are used:

$p=\frac{\rho R T}{\bar{W}}$ and $h_{k}=h_{k}^{o}\left(T_{o}\right)+\int_{T_{o}}^{T} c_{p, k} d T$.

where $R$ is the ideal gas constant, $h_{k}^{o}\left(T_{o}\right)$ is the standard enthalpy of formation of $k$-th gaseous species, $T_{o}$ is the standard ambient temperature of $298.15 \mathrm{~K}$, and $c_{p, k}$ is at the specific heat at constant pressure of $k$-th gaseous species.

Surface species coverage equations:

$\sigma_{m} \frac{\dot{s}_{m}}{\Gamma}=0, \quad m=1, \ldots, M_{s}$.

where $\sigma_{m}$ and $\dot{S}_{m}$ are the site occupancy and heterogeneous molar production rate of $m$-th surface species respectively, $\Gamma$ is the surface site density, and $M_{s}$ is the total number of surface species.

Since the heat transfer along the wall strongly affects the flame stability (Ahn et al., 2005; Federici et al., 2008), the heat conduction within the wall is considered and the energy equation is given as follows:

$\frac{\partial}{\partial x}\left(\lambda_{s} \frac{\partial T}{\partial x}\right)+\frac{\partial}{\partial y}\left(\lambda_{s} \frac{\partial T}{\partial y}\right)=0$.

where $\lambda_{s}$ is the thermal conductivity of the solid wall. Unless otherwise stated, the value of thermal conductivity of the solid wall is set as 16 $\mathrm{W} /(\mathrm{m} \cdot \mathrm{K})$, referring to $\mathrm{FeCr}$-alloy which is a common material for 
catalytic honeycomb combustors in power generation systems (Schneider et al., 2007).

\subsection{Chemical kinetics}

It has been demonstrated that homogeneous chemistry strongly affect the flame stability of catalytic micro-combustors, and the contribution of homogeneous reactions cannot be ignored (Karagiannidis et al., 2007; Karagiannidis et al., 2011). Therefore, the catalytic combustion of fuelair mixtures over platinum is modeled using elementary homogeneous and heterogeneous reaction schemes.

For the homogeneous chemistry of hydrogen, the $\mathrm{H}_{2} / \mathrm{O}_{2}$ mechanism from Burke et al. (2012) is used with its accompanying gas phase thermodynamic data, consisting of 21 reversible elementary reactions with 9 species. For the heterogeneous chemistry of hydrogen, the detailed heterogeneous reaction scheme of Deutschmann et al. (2000) is employed, consisting of 11 irreversible and 3 reversible elementary reactions with 6 gaseous and 5 surface species. The above homogeneous and heterogeneous reaction mechanisms have reproduced the measured catalytic conversions and the homogeneous ignition distances in fuel-lean and fuel-rich hydrogen-air mixtures over platinum (Schultze et al., 2013; Sui et al., 2016).

For the homogeneous chemistry of methane, the detailed homogeneous reaction scheme of GRI-Mech 3.0 (Smith et al., 2000) is employed, consisting of 131 reversible and 6 irreversible elementary reactions with 26 species. For the heterogeneous chemistry of methane, the detailed heterogeneous reaction scheme of Deutschmann et al. (2000) is employed, consisting of 24 elementary reactions with 9 gaseous and 11 surface species.

A surface site density $\Gamma_{\mathrm{Pt}}=2.72 \times 10^{-9} \mathrm{~mol} / \mathrm{cm}^{2}$ is used for the platinum catalyst (Deutschmann et al., 2000). The computational tool DETCHEM (Deutschmann et al., 2014) is applied to treat the problem numerically. Gaseous and surface thermodynamic data are included in the provided schemes. Transport properties are computed from the CHEMKIN transport database (Kee et al., 1998). Homogeneous and heterogeneous reaction rates are evaluated with CHEMKIN (Kee et al., 1996) and Surface-CHEMKIN (Coltrin et al., 1996), respectively.

\subsection{Computation scheme}

The boundary conditions used in this model are as follows. At the inlet, uniform profiles are used for temperature, axial velocity and species mass fractions. To minimize the computational intensity, symmetry boundary conditions are applied at the centerline between the two plates, and half of the system is modeled. At the exit, a fixed pressure is specified and far-field conditions are imposed for the rest of the variables. No-slip is applied for both velocity components at the fluidsolid interface and zero-Neumann conditions are set at the outlet and the plane of symmetry. The heat flux at the fluid-solid interface is computed using Fourier's law and the continuity in temperature and heat flux is ensured. The gas-phase species interfacial boundary conditions are given as follows:

$\left(\rho Y_{k} V_{k, y}\right)_{y=\frac{d}{2}}+W_{k}\left(\dot{s}_{k}\right)_{y=\frac{d}{2}}=0, k=1, \ldots, K_{g}$.

The energy boundary conditions at the fluid-solid interface are given as follows:

$\dot{q}_{r a d}-\lambda_{g}\left(\frac{\partial T}{\partial y}\right)_{y=\frac{d}{2}-}+\lambda_{s}\left(\frac{\partial T}{\partial y}\right)_{y=\frac{d}{2}+}+\sum_{k=1}^{K_{g}}\left(\dot{s}_{k} h_{k} W_{k}\right)=0$.

where the subscripts $(-)$ and $(+)$ denote the properties just below and above the fluid-solid interface, respectively. The term $\dot{q}_{\text {rad }}$ accounts

for radiation exchange of each channel surface element with all other channel surface elements and also with the channel inlet and outlet. The net radiation method for diffuse-gray areas is employed to compute the radiation exchange between the discretized channel wall surface elements themselves and between each surface element and the inlet and outlet channel enclosures. Gas radiative emission and absorption are not considered given the small optical paths and the large nitrogen dilution. Solid-phase radiative boundary conditions are applied at the inlet and outlet vertical wall faces, allowing for heat losses towards the surroundings. All discretized surface elements have a constant emissivity $\varepsilon=0.6$, while the inlet and outlet sections of the radiation enclosure are treated as black bodies (Karagiannidis et al., 2010).

At the exterior surface of the wall, the total heat loss including radiative and convective cooling is taken into account:

$q=h_{o}\left(T_{w, o}-T_{a m b}\right)+\varepsilon_{o} \sigma\left(T_{w, o}^{4}-T_{a m b}^{4}\right)$.

where $q$ is the heat flux; $h_{o}$ is the exterior convective heat transfer coefficient and is assumed to be $20 \mathrm{~W} /\left(\mathrm{m}^{2} \cdot \mathrm{K}\right.$ ) (Liu et al., 2016; Wan et al., 2015), a value in the range of free convection; $T_{w, o}$ is the temperature at the exterior surface; $T_{a m b}$ is the ambient temperature and is assumed to be $300 \mathrm{~K} ; \varepsilon_{o}$ is the exterior surface emissivity and is set as $0.8 ; \sigma$ is the Stephan-Boltzmann constant.

An orthogonal staggered mesh of $200 \times 80$ points (in the $x$ - and $y$ direction, respectively) over half the gas-phase domain is used with finer spacing towards the entry and the wall. The computationally lessintensive solid heat conduction is solved on a $200 \times 20$ grid. Grid independence test has been performed and the final grid density is determined when the centerline temperature and species profiles do not show obvious difference. The fluid viscosity, specific heat, and thermal conductivity are calculated from a mass fraction weighted average of species properties, and the species specific heat is calculated using a piecewise polynomial fit of temperature. The species viscosities and thermal conductivity are determined from kinetic theory. Multicomponent diffusion is considered in this system, and the binary diffusion coefficients are determined from kinetic theory. Homogeneous and heterogeneous chemistries are coupled through the interfacial boundary conditions. The second-order upwind scheme is used to discretize the mathematical model, and the "SIMPLE" correction algorithm is employed to solve for the pressure-velocity coupling (Patankar and Spalding, 1972). The simulation convergence is decided when the residuals of all governing equations approach steady states. With the convergent criteria, the results reported in this work are achieved with the residuals smaller than $1.0 \times 10^{-6}$.

\subsection{Numerical validation}

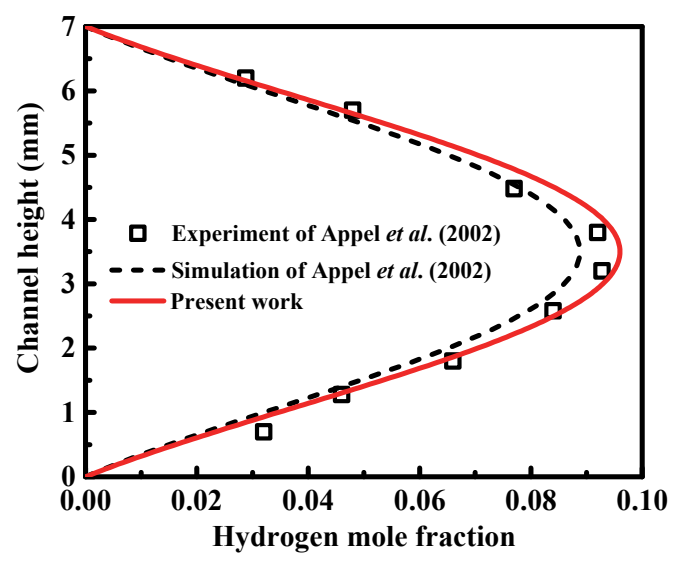

Fig. 2 Comparison of hydrogen mole fraction profiles at the streamwise location of $25 \mathrm{~mm}$ with experimental and numerical results of Appel et al. (2002).

The numerical mode is validated by comparing the experimental and numerical results. Premixed fuel-lean hydrogen-air mixtures at equivalence ratio $\varphi=0.28$ is chosen corresponding to the experimental conditions of Appel et al. (2002). The height and total length of the channel-flow catalytic combustor are $7 \mathrm{~mm}$ and $250 \mathrm{~mm}$, respectively; the inlet temperature is fixed at $312 \mathrm{~K}$ and the inlet velocity is kept at 2 $\mathrm{m} / \mathrm{s}$. The thermocouple-measured surface temperature distributions illustrated in Appel et al. (2002) are used as the interfacial boundary conditions for the temperature when validating the numerical mode. The details of the experimental method and system can be found 
elsewhere (Appel et al. 2002). The Reynolds number (1389) is based on the inlet properties and the channel hydraulic diameter $(13.1 \mathrm{~mm})$, resulting in a laminar flow in the channel.

Fig. 2 shows the transverse mole fraction profiles of fuel at the streamwise location of $25 \mathrm{~mm}$. It can clearly be seen that the numerical predictions are in good agreement with the experimental results. Fig. 3 shows the transverse temperature profiles at the same location, compared to experimental data and numerical predictions reported by Appel et al. (2002). As observed, the temperature field prediction is also in good agreement with the experimental results. These demonstrate that numerical model and experiment results correspond well, and consequently confirm the reasonable accuracy of the numerical model adopted in the present work.

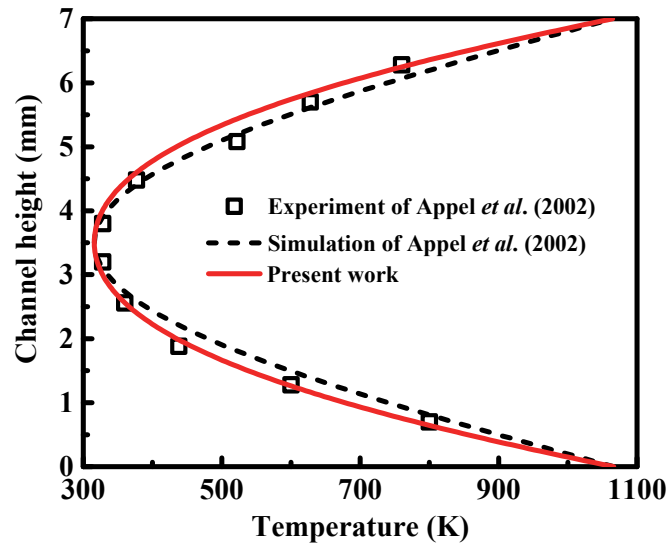

Fig. 3 Comparison of transverse temperature profiles at the streamwise location of $25 \mathrm{~mm}$ with experimental and numerical results of Appel et al. (2002).

\section{RESULTS AND DISCUSSION}

\subsection{Combustion characteristics at the micro-scale}

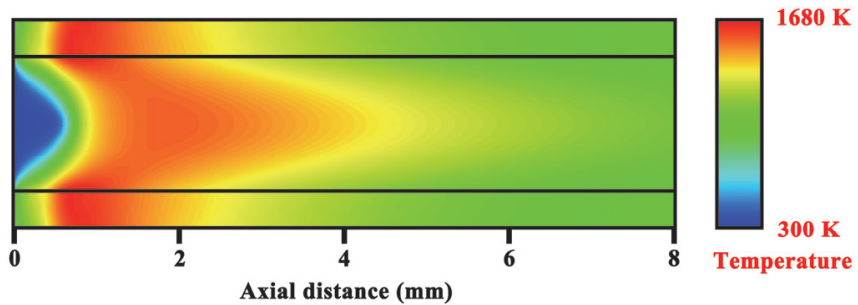

(a) Temperature

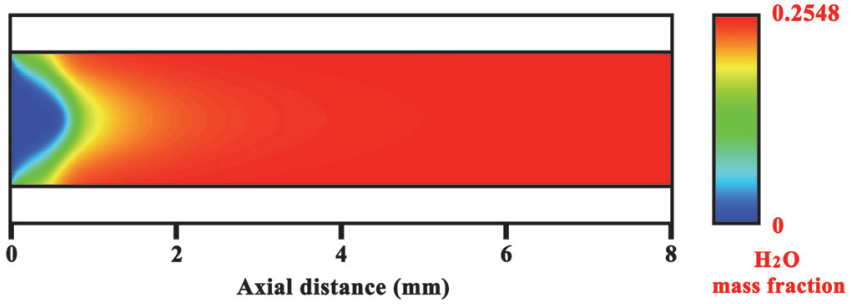

(b) Water mass fraction

Fig. 4 Contour plots of the temperature and water mass fraction for the catalytic combustion of hydrogen-air mixtures. The parameters used are $u_{\text {in }}=0.8 \mathrm{~m} / \mathrm{s}, \lambda_{s}=0.8 \mathrm{~W} /(\mathrm{m} \cdot \mathrm{K}), h_{o}=20 \mathrm{~W} /\left(\mathrm{m}^{2} \cdot \mathrm{K}\right)$, and a stoichiometric feed. Note that the $y$ dimension is scaled by factors of 2 and the reflection of symmetry is used for ease of visualization.

For most cases studied, catalytic micro-combustors exhibit similar combustion characteristics that are summarized in this section. Fig. 4 shows contour plots of the temperature and water mass fraction for the catalytic combustion of stoichiometric hydrogen-air mixtures for a typical set of operating parameters. The inlet velocity $u_{\text {in }}$ is kept constant at $0.8 \mathrm{~m} / \mathrm{s}$. Fig. 5 shows the corresponding temperature and conversion profiles along the wall and fluid centerline. Typically, homogeneous micro-combustors exhibit three regions, namely preheating, combustion, and post-combustion or cooling regions. While these regions are clearly demarcated in homogeneous micro-combustors, the preheating and reaction regions often overlap in catalytic microcombustors. The combustor walls act as a net heat source in the preheating/combustion region because of the axial heat recirculation through wall conduction of the heat released by catalytic combustion, and as a net heat sink in the post-combustion region because of heat losses to the surroundings. Therefore, the wall serves a competing role in flame stability, walls transfer heat upstream for ignition of the cold incoming gases but at the same time are responsible for heat losses. This is consistent with homogeneous micro-combustors (Kaisare et al., 2007a; Norton et al., 2003). The transition in the wall role is also demarcated at the crossover between the wall and bulk gas temperatures.

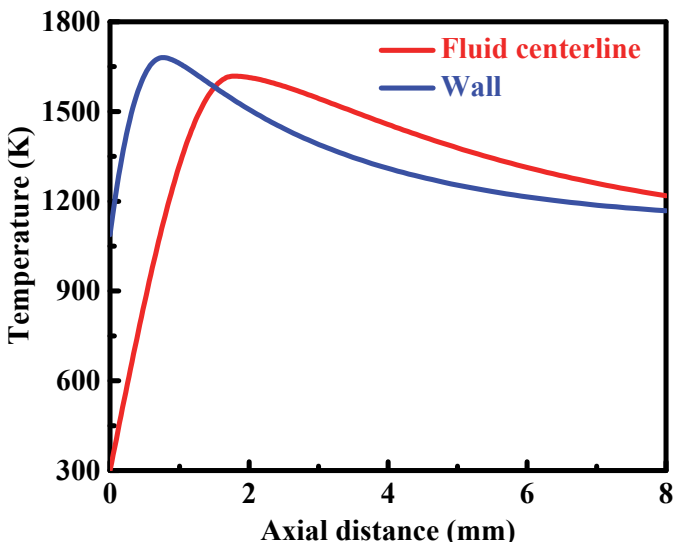

(a) Temperature

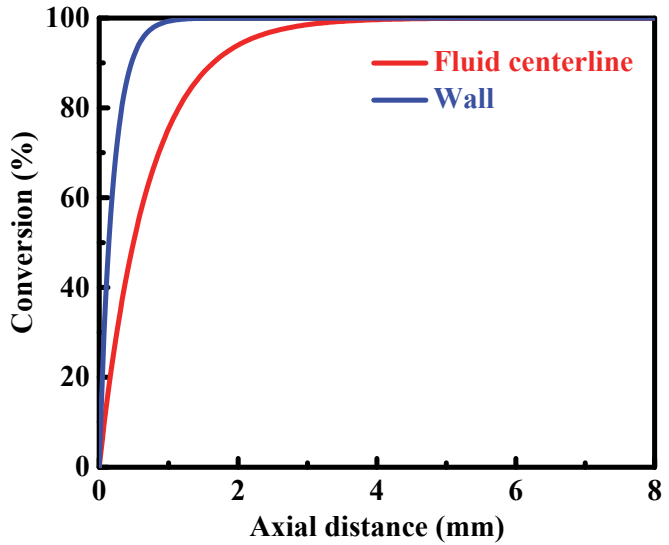

(b) Conversion

Fig. 5 Temperature and conversion profiles along the wall and fluid centerline for the catalytic combustion of hydrogen-air mixtures.

Homogeneous ignition occurs near the catalytically active surfaces and the homogeneous flame travels towards the centerline as the combustible stream flows downstream. An important aspect of the catalytic combustion of hydrogen-air mixtures over platinum catalysts is that the reaction is extremely fast, consuming most of the hydrogen in a very small region due to its low activation energy. Complete conversion is achieved. Despite the small scale involved, mass-transfer limitations are observed within most of the reaction region, which is typical of catalytic combustion. It is expected that further reduction of the gap size could increase the rate of combustion reaction, and could diminish the importance of fuel transport properties because of the shorter characteristic transverse diffusion time scales. These have been 
demonstrated in the numerical and experimental results reported in literatures (Karagiannidis et al., 2011; Norton et al., 2004). A significant temperature rise is observed at the entry, and the homogeneous flame anchors directly at the hot entrance. Despite the small scale, there are significant gradients in temperature and species mass fraction within the fluid near the reaction region, and large axial gradients in temperature exist within the walls. On the other hand, there are no significant transverse gradients within the walls in all cases studied, i.e., the wall is nearly isothermal in the transverse direction. This is due primarily to the short time-scale for conduction within the walls in the transverse direction and the large aspect ratio of the walls. Since there exist axial temperature gradients but near-zero transverse temperature gradients within the walls, only the exterior wall temperature is shown when the wall temperature is displayed in the graphs below.

In the previous work of Merotto et al. (2016), the combustor was coupled with two thermoelectric modules for electric power production. In the previous work of Federici et al. (2006), the combustor was integrated with thermoelectric elements for portable power production. Additionally, thermally conductive metal spreaders were used to create a nearly isothermal interface between the combustor and the thermoelectric device, which allows for improved power generation. In the present work, a stand-alone catalytic micro-combustor without thermoelectric elements is considered. As a result, higher wall temperatures are observed, compared to the experimental data reported in literatures (Federici et al., 2006; Merotto et al., 2016).

\subsection{Effect of wall thermal conductivity}

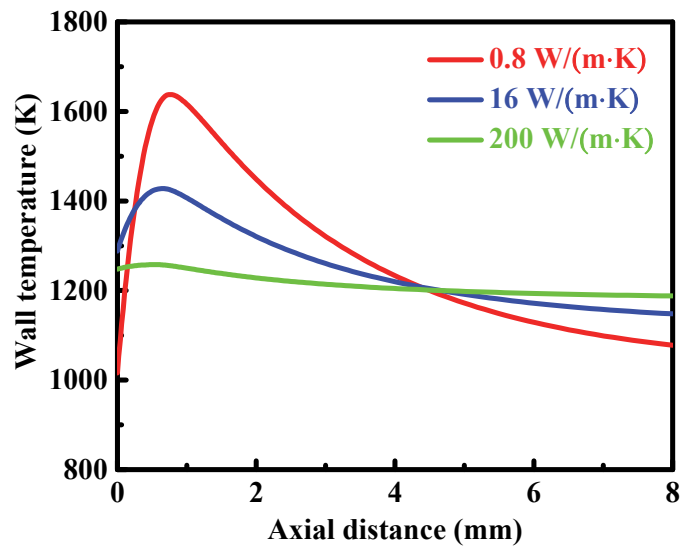

Fig. 6 Axial wall temperature profiles for the catalytic combustion of hydrogen-air mixtures for different wall thermal conductivities. The parameters used are $u_{i n}=0.8 \mathrm{~m} / \mathrm{s}, h_{o}=20 \mathrm{~W} /\left(\mathrm{m}^{2} \cdot \mathrm{K}\right)$, and $\varphi$ $=0.8$.

It has been found that the thermal properties of materials of construction play a vital role in the overall thermal stability of homogeneous micro-combustors, as the combustor walls are responsible for the majority of the upstream heat transfer as well as the external heat losses (Kaisare et al., 2007a; Norton et al., 2003). While homogeneous micro-combustors are feasible to operate, their operation is restrictive. Fig. 6 shows the effect of wall thermal conductivity on the axial wall temperature for the catalytic combustion of hydrogen-air mixtures. The equivalence ratio $\varphi$ is kept constant at 0.8 . Fig. 7 shows the corresponding axial wall temperature profiles for the catalytic combustion of stoichiometric methane-air mixtures. Three wall thermal conductivities of $0.8,16$ and $200 \mathrm{~W} /(\mathrm{m} \cdot \mathrm{K})$ are considered. The first one represents low conductivity ceramics; the second one represents stainless steel, such as FeCr-alloy; the third one represents metals or high conductivity ceramics. It is shown that the wall thermal conductivity has a large effect on the axial wall temperature profile. Very low wall thermal conductivity results in much steeper axial temperature gradients within the wall, with higher downstream wall temperatures, indicating smaller overall heat losses to the surroundings. Hot spots of high temperatures in excess of $1500 \mathrm{~K}$ within the wall can occur, an undesirable situation, as it exceeds the maximum operating temperatures of most materials of construction. Wall and catalyst temperatures in excess of this value are deemed detrimental to the device. For materials with moderate thermal conductivities, on the other hand, axial wall temperature profiles are more uniform, and materials with metallic-like thermal conductivity are practically isothermal. In other words, as the wall thermal conductivity increases, the axial wall temperature profiles become more uniform and the wall hot spot is eliminated. Although the wall is essentially isothermal in the transverse direction for all cases, high wall thermal conductivities greatly reduce the axial wall temperature gradients, regulating the maximum wall temperature. It is expected that the maximum and exit temperature increases with the increase of inlet velocity or with the decrease of exterior convective heat transfer coefficient due to the increased heat input to heat loss ratios. The maximum or exit temperatures are much lower than the adiabatic flame temperatures, given that the adiabatic flame temperatures for stoichiometric hydrogen-air and methane-air mixtures are 2527 and $2236 \mathrm{~K}$, respectively. This is due primarily to the heat losses from the combustor to the surroundings. Improvements over a single micro-channel used here are expected, for example, by heat recirculation, but are beyond the scope of present work. Furthermore, the temperatures are lower than those of homogeneous counterparts (Kaisare et al., 2007a; Norton et al., 2003), which could ensure material stability without hot spots, simplify packaging, and eliminate the possibility of thermal nitrogen oxides formation.

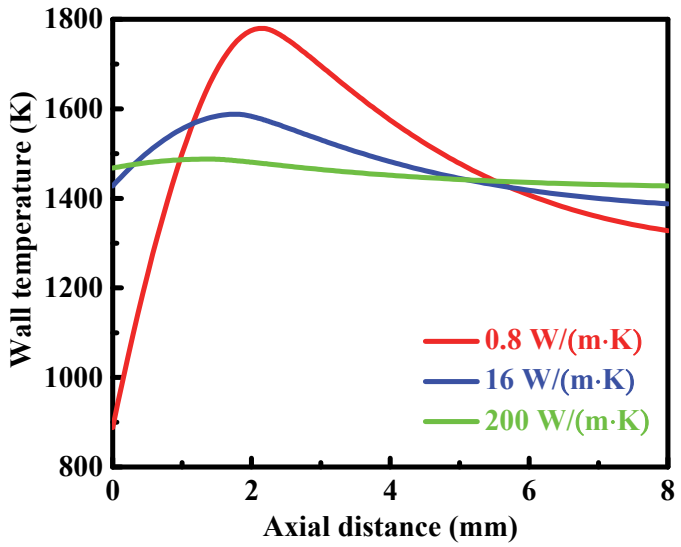

Fig. 7 Axial wall temperature profiles for the catalytic combustion of methane-air mixtures for different wall thermal conductivities. The parameters used are $u_{\text {in }}=0.8 \mathrm{~m} / \mathrm{s}, h_{o}=20 \mathrm{~W} /\left(\mathrm{m}^{2} \cdot \mathrm{K}\right)$, and a stoichiometric feed.

\subsection{Effect of equivalence ratio}

Hydrogen-air mixtures are known to be self-igniting over platinum foils and wires under very fuel-lean conditions (Fassihi et al., 1993; Rinnemo et al., 1993) but fuel-richer mixtures exhibit the ignition temperature above room temperature. Fig. 8 shows the effect of equivalence ratio on the maximum wall temperature and conversion for the catalytic combustion of hydrogen-air mixtures for different wall thermal conductivities. Fig. 9 shows the corresponding temperature and conversion profiles for the catalytic combustion of methane-air mixtures. It is shown that there are a number of important differences between hydrogen-air and methane-air catalytic combustion. Hydrogenair mixtures are found to be self-igniting because of their extremely fast reaction and low activation energy. This property can be used for igniting other hydrocarbons, referred to the hydrogen assisted selfignition of hydrocarbon-air mixtures. It has been demonstrated that hydrogen-air mixtures self-ignite over a wide range of compositions in confined geometry, which can be used to self-ignite propane-air mixtures with the assistance of hydrogen addition (Norton et al., 2005). 
In addition, hydrogen-air mixtures self-ignite and self-sustain over a wide range of compositions, i.e., they exhibit very robust behavior. This is consistent with the experimental result reported in literatures (Norton et al., 2004; Norton et al., 2005), and allows for thermal management to enable the integration of micro-devices for multifunctional tasks. The conversion of hydrogen is complete for all fuel-lean cases but the leanest mixtures. The wall thermal conductivity has little impact on the conversion of hydrogen. For the leanest hydrogen-air mixtures, materials with higher wall thermal conductivities result in slightly lower conversions.

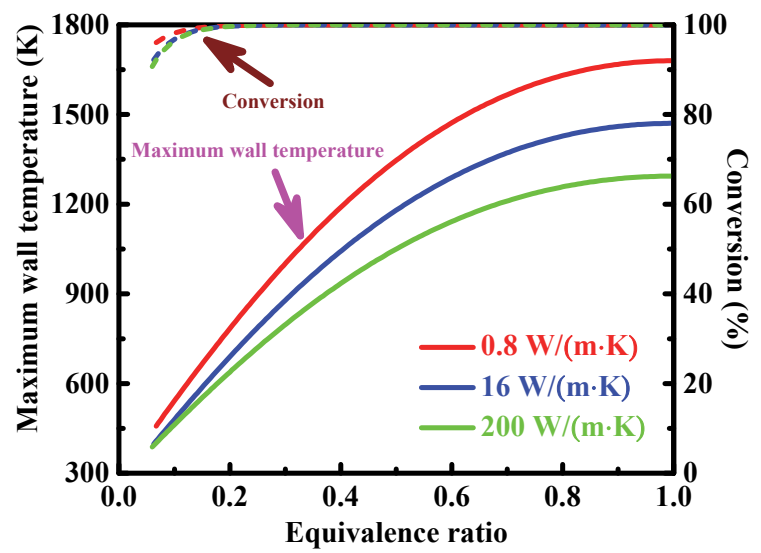

Fig. 8 Maximum wall temperature and conversion as a function of equivalence ratio for the catalytic combustion of hydrogen-air mixtures for different wall thermal conductivities. The parameters used are $u_{\text {in }}=0.8 \mathrm{~m} / \mathrm{s}$ and $h_{o}=20 \mathrm{~W} /\left(\mathrm{m}^{2} \cdot \mathrm{K}\right)$. The solid lines represent the maximum wall temperature, whereas dashed lines represent the conversion.

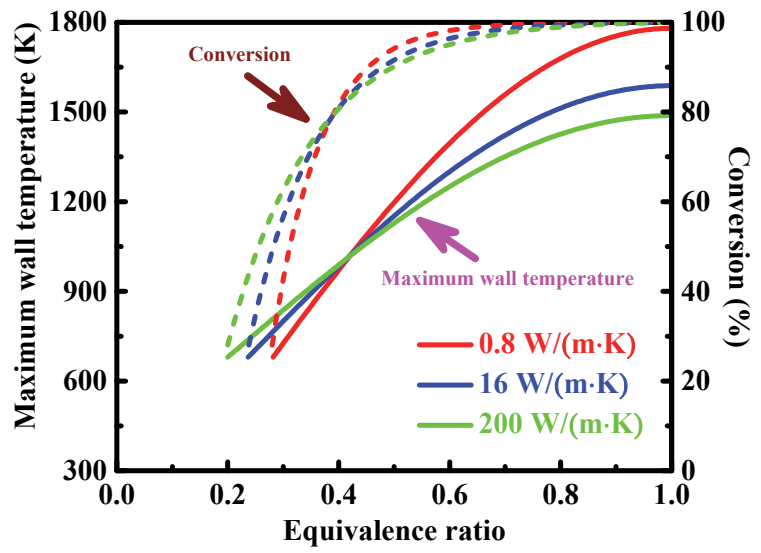

Fig. 9 Maximum wall temperature and conversion as a function of equivalence ratio for the catalytic combustion of methane-air mixtures for different wall thermal conductivities. The parameters used are $u_{i n}=0.8 \mathrm{~m} / \mathrm{s}$ and $h_{o}=20 \mathrm{~W} /\left(\mathrm{m}^{2} \cdot \mathrm{K}\right)$. The solid lines represent the maximum wall temperature, whereas dashed lines represent the conversion.

Unlike hydrogen, methane-air mixtures are not self-igniting at ambient temperature. In contrast to hydrogen, methane is a much heavier molecule, and its adsorption over platinum is activated (Aghalayam et al., 2003; Mhadeshwar et al., 2002), resulting in a more difficult ignition. Therefore, to ignite methane-air mixtures, external heating is necessary. Typically, ignition is achieved by preheating the incoming reacting mixture to a temperature greater than the so-called ignition temperature. On the other hand, the self-ignition nature of hydrogen-air mixtures offers an opportunity to self-ignite hydrocarbons. This concept may be a way toward elimination of ignition sources from micro-devices, resulting in further reduction of system size. Furthermore, since hydrogen is a main target for fuel cell applications, it can be expected that the storage of small amounts of hydrogen during device operation can be obtained from the reforming of hydrocarbons, and can be subsequently used for startup. On the other hand, methaneair mixtures exhibit auto-thermal behavior because they can self-sustain down to an equivalence ratio of approximately 0.2 . For both fuels, the system exhibits highest temperatures near stoichiometric conditions, and the temperature decreases with varying equivalence ratio away from the stoichiometric point because of lower heat generation. While the auto-thermal regime of methane in catalytic micro-combustors is quite large, it is narrower than that of hydrogen. For methane, extinction may occur at low equivalence ratios because the heat released by reaction is insufficient to sustain combustion due to heat loss. At the extinction limit, the ratio of heat losses to the total heat generated is high. Overall, for the same channel size, catalytic microcombustors exhibit much lower temperatures and a wider auto-thermal regime of operation than their homogeneous counterparts (Kaisare et al., 2007a; Norton et al., 2003). Despite the well-known mass transfer limitations of catalytic combustion and the small length of the microchannel, nearly complete conversion of methane is achieved at high equivalence ratios, which is a requirement for high efficiency microsystems. This important result stems from the mere fact that the mass transfer coefficient is high in micro-channels. As the catalytic microcombustor approaches the fuel-lean limit of extinction, both maximum wall temperature and fuel conversion drop considerably with the decrease of fuel composition. However, the wall thermal conductivity has little impact on the extinction limit and conversion. The former is similar to homogeneous micro-combustors in which the wall thermal conductivity has a relatively weaker effect on the extinction limit (Kaisare et al., 2007a; Norton et al., 2003). For both fuels, materials with higher wall thermal conductivities result in lower maximum wall temperatures. This can be attributed to the thermal smoothing associated with the lower thermal resistances of higher wall-thermalconductivity materials, which diffuses the locally generated heat along the length of the combustor wall.

\subsection{Effect of flow rate}

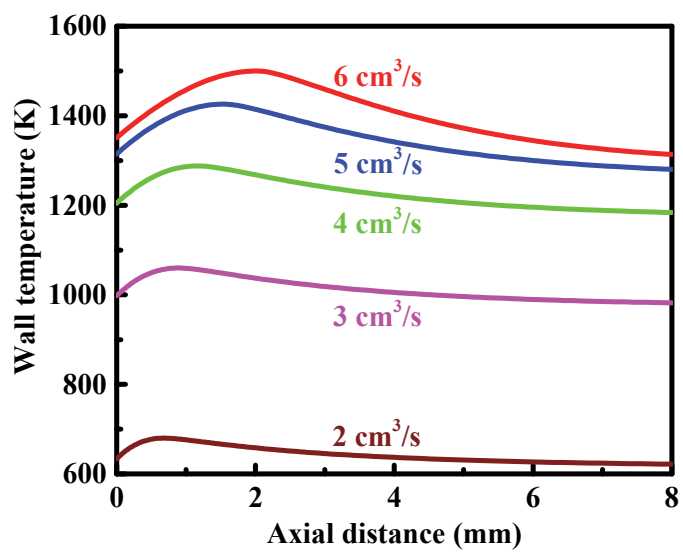

Fig. 10 Axial wall temperature profiles for the catalytic combustion of methane-air mixtures for different flow rates. The parameters used are $\lambda_{s}=16 \mathrm{~W} /(\mathrm{m} \cdot \mathrm{K}), h_{o}=20 \mathrm{~W} /\left(\mathrm{m}^{2} \cdot \mathrm{K}\right)$, and $\varphi=0.8$.

Fig. 10 shows the effect of flow rate on the axial wall temperature for the catalytic combustion of methane-air mixtures. In order to enable a wide range of flow rates, an equivalence ratio of 0.8 is considered. For flow rates below approximately $2 \mathrm{~cm}^{3} / \mathrm{s}$, the catalytic micro-combustor approaches extinction due to excessive heat losses relative to the power generation rate. Under such conditions, the long reactant residence times allow for efficient heat removal from the catalytic microcombustor, resulting in low operating temperatures. As a result, loss of flame stability may occur. The average and maximum wall temperatures increase with the increase of flow rate. This is due primarily to the increased ratio of the heat generation rate to the heat 
loss rate; the former is roughly proportional to the flow rate, whereas the latter is only roughly proportional to the operating temperature. For flow rates above approximately $6 \mathrm{~cm}^{3} / \mathrm{s}$, the maximum wall temperatures exceed the melting temperature of common metal materials. Such high temperatures greatly limit the available materials of construction, result in the production of nitrogen oxides, and require considerable device insulation and packaging. Although temperature reduction is possible by shrinking the device size, self-sustained oscillations in temperature and pressure emerge, eventually resulting in mechanical device failure (Norton et al., 2003). The wall temperature first increases and then decreases with the increase of axial distance. The decrease in wall temperature is due primarily to ambient heat losses. For this rather limited range of flow velocities, the location of the maximum wall temperature does not shift obviously as a function of the flow rate. This implies that the time scales of transport and reaction between the walls and the fluid are much shorter than the residence time.

\subsection{Heat loss}

Generally, micro-combustors could be used to generate heat for utilization by other components or devices. This transfer of thermal energy may occur by various means. The first approach would be to design the micro-combustor itself to directly conduct heat from the reacting gases to another device. For example, micro-combustors are integrated with other components, such as heat exchangers or smallscale fuel processing or reforming systems (Holladay and Wang, 2015; Kim, 2009), for portable power generation systems. In this design, the micro-combustor has partial or minimal insulation, and thermal losses through the walls would be used directly. In another approach, the system would be highly insulated, so that all of the enthalpy would stay within the flowing gases. These heated gases would then could be transferred to another component or could be converted into another energy, such as a turbine, a piston, or a thermoelectric, thermophotovoltaic (TPV) or piezoelectric device, for utilization of the enthalpy of the gases (Ganji and Ebrahimi, 2013; Ohadi and Buckley, 2001).

As mentioned above, the maximum or exit temperatures in catalytic micro-combustors are much lower than the adiabatic flame temperatures. This implies that significant heat is transferred through the combustor walls. To assist the analysis, the concept of fractional enthalpy loss $\eta_{\text {loss }}$ is used. The fractional enthalpy loss is defined as the ratio of the enthalpy loss to the surroundings to the enthalpy loss if the flow reached ambient temperatures.

$\eta_{\text {loss }}=\frac{h\left(T_{\text {in }}, x_{\text {in }}\right)-h\left(T_{\text {out }}, x_{\text {out }}\right)}{h\left(T_{\text {in }}, x_{\text {in }}\right)-h\left(T_{\text {amb }}, x_{\text {out }}\right)}$.

where $T_{\text {in }}$ is the inlet temperature and is assumed to be ambient temperature, $x_{i n}$ is the mole fraction at the intlet of the combustor, and $T_{\text {out }}$ and $x_{\text {out }}$ are the average mole fractions at the outlet of the combustor, respectively. The fractional enthalpy loss could be used to depict in what manner the enthalpy released from the catalytic combustion is transferred from the combustor to a possible adjacent device.

Fig. 11 shows the effect of equivalence ratio on the fractional enthalpy loss and maximum wall temperature difference for the catalytic combustion of hydrogen-air mixtures. The flow rate is kept constant at $6 \mathrm{~cm}^{3} / \mathrm{s}$. By the time the products reach the end of the channel, most of the enthalpy released by the exothermic reaction is lost to the surroundings. These high values of enthalpy loss are observed because micro-combustors inherently have very high surface area-tovolume ratios. This feature is detrimental for the design of adiabatic systems but beneficial for heat-exchanger applications. On the other hand, the thermal non-uniformity slightly increases with the increase of equivalence ratio. This can be attributed to the increase in temperature with the increase of equivalence ratio, resulting in higher reaction rates and thus more localized heat generation. In contrast, fuel-leaner feeds allow lower reaction rates over a broader reaction region, resulting in more uniform temperature profiles.

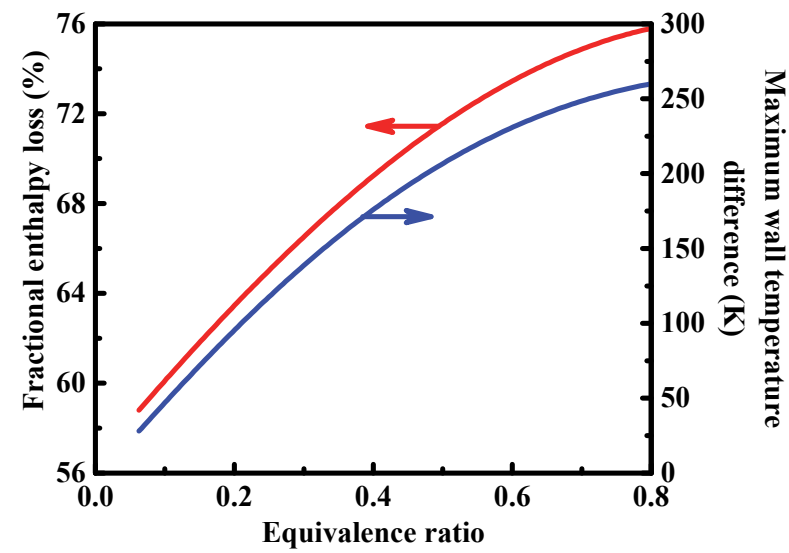

Fig. 11 Effect of equivalence ratio on the fractional enthalpy loss and maximum wall temperature difference for the catalytic combustion of hydrogen-air mixtures. The parameters used are $\lambda_{s}=16 \mathrm{~W} /(\mathrm{m} \cdot \mathrm{K}), h_{o}=20 \mathrm{~W} /\left(\mathrm{m}^{2} \cdot \mathrm{K}\right)$, and $Q=6 \mathrm{~cm}^{3} / \mathrm{s}$.

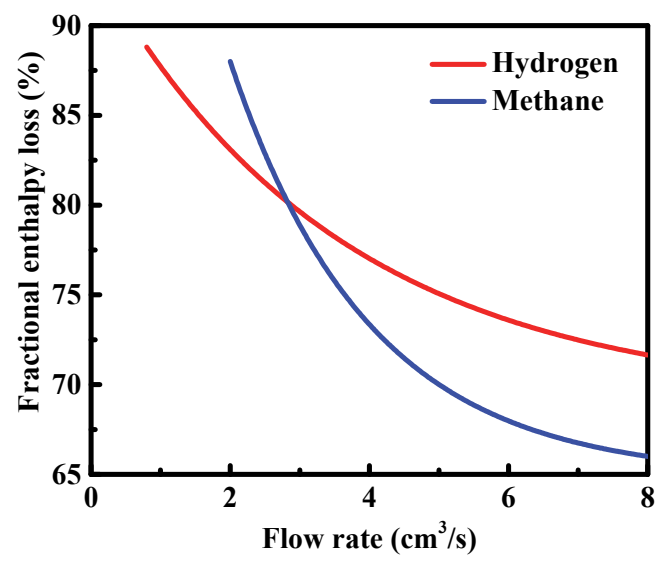

Fig. 12 Fractional enthalpy loss profiles for the catalytic combustion of hydrogen-air and methane-air mixtures for different flow rates. The parameters used are $\lambda_{s}=16 \mathrm{~W} /(\mathrm{m} \cdot \mathrm{K}), h_{o}=20 \mathrm{~W} /\left(\mathrm{m}^{2} \cdot \mathrm{K}\right), \varphi$ (hydrogen-air mixtures) $=0.6$, and $\varphi$ (methane-air mixtures $)=$ 0.8 .

Fig. 12 shows the effect of flow rate on the fractional enthalpy loss for the catalytic combustion of hydrogen-air and methane-air mixtures. The equivalence ratios of hydrogen-air and methane-air mixtures are kept constant at 0.6 and 0.8 , respectively. For both fuels, the fractional enthalpy loss decreases with the increase of flow rate. This behavior is a consequence of more heat generated into the system with the increase of flow rate. As far as complete conversion can be achieved, most of the heat generated by catalytic combustion is carried out as the enthalpy of the fluid rather than is lost to the surroundings. This is due primarily to the decreased residence time. The combination results in less heat loss to the surroundings per unit flow rate of reactant. This is an advantage from the stability point of view but a disadvantage for integrated microdevices. To be specific, if sufficiently fast flows are required and a significant amount of heat is to be harvested from the flows, ample combustor surface area would be necessary to achieve the necessary heat exchange when designing catalytic micro-combustors. Substantial increases in flow rate would eventually result in incomplete conversion, breakthrough, and even blowout. This is due primarily to low residence times associated with high heat losses in the form of a hot exit gas (Kaisare et al., 2007b; Yan et al., 2016). 


\section{CONCLUSIONS}

The combustion and heat transfer characteristics in catalytic microcombustors were studied numerically to investigate the effect of wall thermal conductivity, feed composition, and flow rate on the thermal uniformity. Additionally, an overall energy balance analysis was carried out in terms of enthalpy loss. It was shown that the thermal uniformity is highly dependent on combustor material, feed composition, and flow rate, and can be controlled by these wall thermal properties and key operation parameters. Therefore, the specific design of catalytic microcombustors can be tailored depending on the particular application. For mildly endothermic or exothermic reactions, it may be possible to achieve the desired level of thermal uniformity easily. However, for highly endothermic or exothermic reactions, particular attention should be paid to the temperature range of catalytic micro-combustors to determine whether it is adequate. The major conclusions are summarized as follows:

- The wall thermal conductivity has a significant effect on the thermal uniformity, but has little impact on the extinction limit and conversion.

- The increase of fuel composition results in higher temperatures and greater thermal non-uniformity, but the choice of fuel plays a small role in determining thermal uniformity.

- The flow rate has a moderate effect on the thermal uniformity. Specifically, higher flow rates increase temperatures and reduce thermal uniformity, whereas lower flow rates improve thermal uniformity but may induce extinction due to increased heat loss to heat generation ratios.

- An overall energy balance analysis reveals that most of the enthalpy released by the exothermic reaction is lost to the surroundings under certain conditions because of the high surface area-to-volume ratio, although this energy exchange becomes less efficient as the flow rate increases. This feature is detrimental for the design of adiabatic systems but beneficial for heat-exchanger applications.

\section{ACKNOWLEDGEMENTS}

This work was supported by the National Natural Science Foundation of China (No. 51506048).

\section{REFERENCES}

Aghalayam, P., Park, Y.K., Fernandes, N., Papavassiliou, V., Mhadeshwar, A.B., and Vlachos, D.G., 2003, "A C1 Mechanism for Methane Oxidation on Platinum," Journal of Catalysis, 213(1), 23-38. http://dx.doi.org/10.1016/S0021-9517(02)00045-3

Ahn, J., Eastwood, C., Sitzki, L., and Ronney, P.D., 2005, "Gas-phase and Catalytic Combustion in Heat-Recirculating Burners," Proceedings of the Combustion Institute, 30(2), 2463-2472.

http://dx.doi.org/10.1016/j.proci.2004.08.265

Appel, C., Mantzaras, J., Schaeren, R., Bombach, R., Inauen, A., Kaeppeli, B., Hemmerling, B., and Stampanoni, A., 2002, "An Experimental and Numerical Investigation of Homogeneous Ignition in Catalytically Stabilized Combustion of Hydrogen/Air Mixtures Over Platinum," Combustion and Flame, 128(4), 340-368.

http://dx.doi.org/10.1016/S0010-2180(01)00363-7

Bagheri, G., and Hosseini, S.E., 2015, "Impacts of Inner/Outer Reactor Heat Recirculation on the Characteristic of Micro-Scale Combustion System," Energy Conversion and Management, 105, 45-53. http://dx.doi.org/10.1016/j.enconman.2015.07.056

Boger, T., and Heibel, A.K., 2005, "Heat Transfer in Conductive Monolith Structures," Chemical Engineering Science, 60(7), 1823-1835. http://dx.doi.org/10.1016/j.ces.2004.11.031
Burke, M.P., Chaos, M., Ju, Y., Dryer, F.L., and Klippenstein, S.J., 2012, "Comprehensive $\mathrm{H}_{2} / \mathrm{O}_{2}$ Kinetic Model for High-Pressure Combustion," International Journal of Chemical Kinetics, 44(7), 444474.

http://dx.doi.org/10.1002/kin.20603

Chen, G.-B., Chao, Y.-C., and Chen, C.-P., 2008, "Enhancement of Hydrogen Reaction in a Micro-Channel by Catalyst Segmentation," International Journal of Hydrogen Energy, 33(10), 2586-2595.

http://dx.doi.org/10.1016/j.ijhydene.2008.02.071

Chiuta, S., Everson, R.C., Neomagus, H.W.J.P., Gryp, P. van der, and Bessarabov, D.G., 2013, "Reactor Technology Options for Distributed Hydrogen Generation via Ammonia Decomposition: A Review," International Journal of Hydrogen Energy, 38(35), 14968-14991. http://dx.doi.org/10.1016/j.ijhydene.2013.09.067

Coltrin, M.E., Kee, R.J., Rupley, F.M., and Meeks, E., 1996, SURFACE CHEMKIN-III: A Fortran Package for Analyzing Heterogeneous Chemical Kinetics at a Solid-Surface-Gas-Phase Interface, Report No. SAND96-8217, Sandia National Laboratories.

http://dx.doi.org/10.2172/481906

Deutschmann, O., Maier, L.I., Riedel, U., Stroemman, A.H., and Dibble, R.W., 2000, "Hydrogen Assisted Catalytic Combustion of Methane on Platinum," Catalysis Today, 59(1-2), 141-150.

http://dx.doi.org/10.1016/S0920-5861(00)00279-0

Deutschmann, O., Tischer, S., Correa, C., Chatterjee, D., Kleditzsch, S., Janardhanan, V.M., Mladenov, N., Minh, H.D., Karadeniz, H., and Hettel, M., 2014, DETCHEM Software Package, 2.5 ${ }^{\text {th }}$ ed., Karlsruhe, http://www.www.detchem.com (accessed March 4, 2017).

Di Stazio, A., Chauveau, C., Dayma, G., and Dagaut, P., 2016, "Combustion in Micro-Channels with a Controlled Temperature Gradient," Experimental Thermal and Fluid Science, 73, 79-86. http://dx.doi.org/10.1016/j.expthermflusci.2015.09.020

Fassihi, M., Zhdanov, V.P., Rinnemo, M., Keck, K.E., and Kasemo, B., 1993, "A Theoretical and Experimental Study of Catalytic Ignition in the Hydrogen-Oxygen Reaction on Platinum," Journal of Catalysis, 141(2), 438-452.

http://dx.doi.org/10.1006/jcat.1993.1153

Federici, J.A., and Vlachos, D.G., 2008, "A Computational Fluid Dynamics Study of Propane/Air Microflame Stability in a Heat Recirculation Reactor," Combustion and Flame, 153(1-2), 258-269.

http://dx.doi.org/10.1016/j.combustflame.2007.09.009

Federici, J.A., Norton, D.G., Brüggemann, T., Voit, K.W., Wetzel, E.D., and Vlachos, D.G., 2006, "Catalytic Microcombustors with Integrated Thermoelectric Elements for Portable Power Production," Journal of Power Sources, 161(2), 1469-1478.

http://dx.doi.org/10.1016/j.jpowsour.2006.06.042

Fluent Inc., 2006, Fluent 6.3 User's Guide, Lebanon, New Hampshire.

Ganji, H.B., and Ebrahimi, R., 2013, "Numerical Estimation of Blowout, Flashback, and Flame Position in MIT Micro Gas-Turbine Chamber," Chemical Engineering Science, 104, 857-867.

http://dx.doi.org/10.1016/j.ces.2013.09.056

Groppi G., and Tronconi, E., 2005, "Honeycomb Supports with High Thermal Conductivity for Gas/Solid Chemical Processes," Catalysis Today, 105(3-4), 297-304.

http://dx.doi.org/10.1016/j.cattod.2005.06.041

Haber, J., Kashid, M.N., Renken, A., and Kiwi-Minsker, L., 2012, "Heat Management in Single and Multi-Injection Microstructured Reactors: Scaling Effects, Stability Analysis, and Role of Mixing," Industrial \& Engineering Chemistry Research, 51(4), 1474-1489. http://dx.doi.org/10.1021/ie201158a 
Holladay, J.D., and Wang, Y., 2015, “A Review of Recent Advances in Numerical Simulations of Microscale Fuel Processor for Hydrogen Production," Journal of Power Sources, 282, 602-621. http://dx.doi.org/10.1016/j.jpowsour.2015.01.079

Jeon, S.W., Yoon, W.J., Baek, C., and Kim, Y., 2013, "Minimization of Hot Spot in a Microchannel Reactor for Steam Reforming of Methane with the Stripe Combustion Catalyst Layer," International Journal of Hydrogen Energy, 38(32), 13982-13990.

http://dx.doi.org/10.1016/j.ijhydene.2013.08.074

Jeon, S.W., Yoon, W.J., Jeong, M.W., and Kim, Y., 2014, "Optimization of a Counter-Flow Microchannel Reactor Using Hydrogen Assisted Catalytic Combustion for Steam Reforming of Methane," International Journal of Hydrogen Energy, 39(12), 64706478 .

http://dx.doi.org/10.1016/j.ijhydene.2014.02.012

Ju, Y., and Maruta, K., 2011, "Microscale Combustion: Technology Development and Fundamental Research," Progress in Energy and Combustion Science, 37(6), 669-715.

http://dx.doi.org/10.1016/j.pecs.2011.03.001

Kaisare, N.S., and Vlachos, D.G., 2007a, “Optimal Reactor Dimensions for Homogeneous Combustion in Small Channels," Catalysis Today, 120(1), 96-106.

http://dx.doi.org/10.1016/j.cattod.2006.07.036

Kaisare, N.S., and Vlachos, D.G., 2007b, "Extending the Region of Stable Homogeneous Micro-Combustion Through Forced Unsteady Operation," Proceedings of the Combustion Institute, 31(2), 3293-3300. http://dx.doi.org/10.1016/j.proci.2006.07.031

Kaisare, N.S., and Vlachos, D.G., 2012, "A Review on Microcombustion: Fundamentals, Devices and Applications," Progress in Energy and Combustion Science, 38(3), 321-359.

http://dx.doi.org/10.1016/j.pecs.2012.01.001

Karagiannidis, S., Mantzaras, J., and Boulouchos, K., 2011, "Stability of Hetero-/Homogeneous Combustion in Propane- and Methane-Fueled Catalytic Microreactors: Channel Confinement and Molecular Transport Effects," Proceedings of the Combustion Institute, 33(2), 3241-3249.

http://dx.doi.org/10.1016/j.proci.2010.05.107

Karagiannidis, S., Mantzaras, J., Jackson, G., and Boulouchos, K., 2007, "Hetero-/Homogeneous Combustion and Stability Maps in MethaneFueled Catalytic Microreactors," Proceedings of the Combustion Institute, 31(2), 3309-3317.

http://dx.doi.org/10.1016/j.proci.2006.07.121

Karagiannidis, S., Marketos, K., Mantzaras, J., Schaeren, R., and Boulouchos, K., 2010, "Experimental and Numerical Investigation of a Propane-Fueled, Catalytic Mesoscale Combustor," Catalysis Today, 155(1-2), 108-115.

http://dx.doi.org/10.1016/j.cattod.2010.04.030

Kee, R.J., Dixon-lewis, G., Warnatz, J., Coltrin, M.E., Miller, J.A., and Moffat, H.K., 1998, A Fortran Computer Code Package for the Evaluation of Gas-Phase, Multicomponent Transport Properties, Report No. SAND86-8246B, Sandia National Laboratories.

Kee, R.J., Rupley, F.M., Meeks, E., and Miller, J.A., 1996, CHEMKINIII: A Fortran Chemical Kinetics Package for the Analysis of Gasphase Chemical and Plasma Kinetics, Report No. SAND96-8216, Sandia National Laboratories.

http://dx.doi.org/10.2172/481621

Kim, N.I., Aizumi, S., Yokomori, T., Kato, S., Fujimori, T., and Maruta, K., 2007, "Development and Scale Effects of Small Swiss-Roll Combustors," Proceedings of the Combustion Institute, 31(2), 32433250 . http://dx.doi.org/10.1016/i.proci.2006.08.077

Kim, N.I., Kato, S., Kataoka, T., Yokomori, T., Maruyama, S., Fujimori, T., and Maruta, K., 2005, "Flame Stabilization and Emission of Small Swiss-Roll Combustors as Heaters," Combustion and Flame, 141(3), 229-240.

http://dx.doi.org/10.1016/j.combustflame.2005.01.006

Kim, T., 2009, "Micro Methanol Reformer Combined with a Catalytic Combustor for a PEM Fuel Cell," International Journal of Hydrogen Energy, 34(16), 6790-6798.

http://dx.doi.org/10.1016/j.ijhydene.2009.06.024

Liu, Y., Fan, A., Yao, H., and Liu, W., 2016, “A Numerical Investigation on the Effect of Wall Thermal Conductivity on Flame Stability and Combustion Efficiency in a Mesoscale Channel Filled with Fibrous Porous Medium," Applied Thermal Engineering, 101, 239-246.

http://dx.doi.org/10.1016/j.applthermaleng.2016.02.099

Merotto, L., Fanciulli, C., Dondè, R., and De Iuliis, S., 2016, "Study of a Thermoelectric Generator Based on a Catalytic Premixed Meso-Scale Combustor," Applied Energy, 162, 346-353.

http://dx.doi.org/10.1016/j.apenergy.2015.10.079

Mhadeshwar, A.B., Aghalayam, P., Papavassiliou, V., and Vlachos, D.G., 2002, "Surface Reaction Mechanism Development for PlatinumCatalyzed Oxidation of Methane," Proceedings of the Combustion Institute, 29(1), 997-1004.

http://dx.doi.org/10.1016/S1540-7489(02)80126-2

Miesse, C.M., Masel, R.I., Jensen, C.D., Shannon, M.A., and Short, M., 2004, "Submillimeter-Scale Combustion," AIChE Journal, 50(12), 3206-3214.

http://dx.doi.org/10.1002/aic.10271

Norton, D.G., and Vlachos, D.G., 2003, "Combustion Characteristics and Flame Stability at the Microscale: A CFD Study of Premixed Methane/Air Mixtures," Chemical Engineering Science, 58(21), 48714882.

http://dx.doi.org/10.1016/j.ces.2002.12.005

Norton, D.G., and Vlachos, D.G., 2005, "Hydrogen Assisted SelfIgnition of Propane/Air Mixtures in Catalytic Microburners," Proceedings of the Combustion Institute, 30(2), 2473-2480.

http://dx.doi.org/10.1016/j.proci.2004.08.188

Norton, D.G., Wetzel, E.D., and Vlachos, D.G., 2004, "Fabrication of Single-Channel Catalytic Microburners: Effect of Confinement on the Oxidation of Hydrogen/Air Mixtures," Industrial \& Engineering Chemistry Research, 43(16), 4833-4840.

http://dx.doi.org/10.1021/ie049798b

Norton, D.G., Wetzel, E.D., and Vlachos, D.G., 2006, "Thermal Management in Catalytic Microreactors," Industrial \& Engineering Chemistry Research, 45(1), 76-84.

http://dx.doi.org/10.1021/ie050674o

Ohadi, M.M., and Buckley, S.G., 2001, "High Temperature Heat Exchangers and Microscale Combustion Systems: Applications to Thermal System Miniaturization," Experimental Thermal and Fluid Science, 25(5), 207-217.

http://dx.doi.org/10.1016/S0894-1777(01)00069-3

Patankar, S.V., and Spalding, D.B., 1972, “A Calculation Procedure for Heat, Mass and Momentum Transfer in Three-Dimensional Parabolic Flows," International Journal of Heat and Mass Transfer, 15(10), 1787-1806.

http://dx.doi.org/10.1016/0017-9310(72)90054-3

Rana, U., Chakraborty, S., and Som, S.K., 2014, "Thermodynamics of Premixed Combustion in a Heat Recirculating Micro Combustor," Energy, 68, 510-518. 


\section{http://dx.doi.org/10.1016/j.energy.2014.02.070}

Rinnemo, M., Fassihi, M., and Kasemo, B., 1993, "The Critical Condition for Catalytic Ignition. $\mathrm{H}_{2} / \mathrm{O}_{2}$ on Pt," Chemical Physics Letters, 211(1), 60-64.

http://dx.doi.org/10.1016/0009-2614(93)80052-Q

Schneider, A., Mantzaras, J., and Eriksson, S., 2007, "Ignition and Extinction in Catalytic Partial Oxidation of Methane-Oxygen Mixtures with Large $\mathrm{H}_{2} \mathrm{O}$ and $\mathrm{CO}_{2}$ Dilution," Combustion Science and Technology, 180(1), 89-126.

http://dx.doi.org/10.1080/00102200701487087

Schultze, M., Mantzaras, J., Bombach, R., and Boulouchos, K., 2013, "An Experimental and Numerical Investigation of the Hetero/Homogeneous Combustion of Fuel-Rich Hydrogen/Air Mixtures Over Platinum," Proceedings of the Combustion Institute, 34(2), 2269-2277. http://dx.doi.org/10.1016/j.proci.2012.05.029

Shigarov, A.B., Fadeev, S.I., and Kirillov, V.A., 2009, "Modeling of a Heat-Integrated Catalytic Reformer/Combustor of Methane: Fine Balancing Between Hot Spots and Extinction," Chemical Engineering \& Technology, 32(9), 1367-1375.

http://dx.doi.org/10.1002/ceat.200900197

Shirsat, V., and Gupta, A.K., 2011, "A Review of Progress in Heat Recirculating Meso-Scale Combustors," Applied Energy, 88(12), 42944309.

http://dx.doi.org/10.1016/j.apenergy.2011.07.021

Smith, G.P., Golden, D.M., Frenklach, M., Moriarty, N.W., Eiteneer, B., Goldenberg, M., Bowman, C.T., Hanson, R.K., Song, S., Gardiner, Jr., W.C., Lissianski, V.V., and Qin, Z., 2000, GRI-Mech 3.0, Gas Research
Institute, http://www.me.berkeley.edu/gri-mech/ (accessed March 4, 2017).

Sui, R., Prasianakis, N.I., Mantzaras, J., Mallya, N., Theile, J., Lagrange, D., and Friess, M., 2016, "An Experimental and Numerical Investigation of the Combustion and Heat Transfer Characteristics of Hydrogen-Fueled Catalytic Microreactors," Chemical Engineering Science, 141, 214-230.

http://dx.doi.org/10.1016/i.ces.2015.10.034

Tolmachoff, E.D., Booth, A.D., Lee, I.C., Allmon, W.R., and Waits, C.M., 2015, "Modeling and Experimental Analysis of $N$-Dodecane Oxidation in Platinum-Coated Channels," Combustion and Flame, 162(10), 3674-3680.

http://dx.doi.org/10.1016/j.combustflame.2015.07.006

Walther, D.C., and Ahn, J., 2011, "Advances and Challenges in the Development of Power-Generation Systems at Small Scales," Progress in Energy and Combustion Science, 37(5), 583-610.

http://dx.doi.org/10.1016/j.pecs.2010.12.002

Wan, J., Fan, A., Liu, Y., Yao, H., Liu, W., Gou, X., and Zhao, D., 2015, "Experimental Investigation and Numerical Analysis on Flame Stabilization of $\mathrm{CH}_{4} /$ Air Mixture in a Mesoscale Channel with Wall Cavities," Combustion and Flame, 162(4), 1035-1045. http://dx.doi.org/10.1016/j.combustflame.2014.09.024

Yan, Y., Huang, W., Tang, W., Zhang, L., Li, L., Ran, J., and Yang, Z., 2016, "Numerical Study on Catalytic Combustion and Extinction Characteristics of Pre-Mixed Methane-Air in Micro Flatbed Channel Under Different Parameters of Operation and Wall," Fuel, 180, 659667.

http://dx.doi.org/10.1016/j.fuel.2016.04.085 